\title{
Relationship Between Physical Fitness Variables and Reaction Time in eSports Gamers
}

\author{
Rachel Dykstra, Nebraska Wesleyan University, USA \\ Panagiotis Koutakis, Baylor University, USA \\ (iD https://orcid.org/0000-0002-8352-5667 \\ Nicholas Hanson, Western Michigan University, USA
}

\begin{abstract}
eSports gaming requires quick and accurate reactions in response to complex visual stimuli for optimal performance. The purpose of this study was to examine the relationship between components of physical fitness and reaction time (RT) in eSports gamers. Twenty-seven eSports gamers participated. Visit 1 included physical fitness assessments. Visit 2 included a familiarization with the Stroop Task. During Visit 3, subjects completed the Stroop Task again; data from this visit was used for analyses. For congruent, incongruent, and control trials, an inverse efficiency score (IES) was calculated. Pearson correlations were used to determine the relationship between components of physical fitness and mean RT. There was a significant correlation between mean RT and cardiorespiratory fitness $(\mathrm{r}=-.338, \mathrm{p}=.043)$. IES was significantly related to cardiorespiratory fitness in incongruent $(\mathrm{r}=-.373$, $\mathrm{p}=.028)$ and control $(\mathrm{r}=-.333, \mathrm{p}=.045)$ trials. Results suggest that increased cardiorespiratory fitness can improve reaction time and also induce greater efficiency.
\end{abstract}

\section{KEYWORDS}

Aerobic Exercise, Cardiorespiratory Fitness, Cognitive Function, Competitive Gaming, Inverse Efficiency Score, Public Health, Stroop Task, Video Gaming

\section{INTRODUCTION}

Electronic sports, or more commonly called "eSports," refers to competitive video gaming (e.g. leagues and tournaments) facilitated by electronic systems (Hamari \& Sjöblom, 2017). With over 335 million playing and actively spectating year round (Hamari \& Sjöblom, 2017), eSports has developed into one of the fastest growing forms of entertainment in the media. This has shifted the perception of video gaming from a leisurely activity to an organized professional sport (Hallmann \& Giel, 2018). Correspondingly, research on video gaming has evolved; early research depicted video gaming as potentially addictive (Bediou et al., 2018) and psychologically harmful (Pallavicini, Ferrari, \& Mantovani, 2018), but now studies have recognized that it has the potential to positively impact various physiological (Kari, Siutila, \& Karhulahti, 2019; Polman, Trotter, Poulus, \& Borkoles, 2018), sociological (Seo \& Jung, 2016) and psychological factors (Bediou et al., 2018; Bányai, Griffiths, Király, \& Demetrovics, 2019; Pallavicini et al., 2018). As a result, researchers in many fields have a

\section{DOI: 10.4018/IJER.288540}

This article published as an Open Access article distributed under the terms of the Creative Commons Attribution License (http://creativecommons.org/licenses/by/4.0/) which permits unrestricted use, distribution, and production in any medium, provided the author of the original work and original publication source are properly credited. 
considerable interest in determining if there are any connections between competitive video gaming and variables such as cognitive function and physical fitness (Pallavicini et al., 2018; Perrin et al., 2019; Polman et al., 2018).

Competitive video gaming puts a large emphasis on cognitive demand (Dye, Green, \& Bavelier, 2009; Wang et al., 2016). Competitive video (eSports) gamers are required to rapidly process information, and react quickly and accurately in response to complex visual stimuli for optimal gaming performance (Campbell, Toth, Moran, Kowal, \& Exton, 2018; Dye et al., 2009). Thus, eSports gamers have reason to be motivated to improve cognitive function. Reaction time (RT), or the time interval between a stimulus and the response to it (Radák, 2018), plays a vital role for eSports gamers (Bediou et al., 2018; Pallavicini et al., 2018); delays in RT and processing speed often generate negative consequences during gaming (e.g. less competitive advantage, lower percentage of game wins, etc.) (Dye et al., 2009). Moreover, eSports players have determined that training reaction time is rather important, as fast RT is a constituent for success in most competitive games (Nagorsky \& Wiemeyer, 2020). Previous research has revealed that deficits in RT are associated with impaired cognitive ability and serve as valuable measures for establishing cognitive efficiency, the brain's ability to perform optimally with minimal effort (Collins \& Long, 1996).

Preceding studies have demonstrated that aerobic fitness training augments cognitive function (Colcombe \& Kramer, 2003). In a meta-analysis (Smith et al., 2010), the effects of aerobic training programs on cognitive function was assessed. For all training program durations [e.g., short (1-3 months), moderate (4-6 months), and long ( $>6$ months)] with various session durations [e.g., short (15-30 minutes), moderate (31-45 minutes), and long (45-60 minutes)], significant improvements in RT were reported. Significant improvements in the meta-analysis were observed irrespective of exercise duration and intensity and age of subjects (Smith et al., 2010). Subsequent research has since been conducted to corroborate these results. Verma, Mishra, and Singh (Verma, Mishra, \& Singh, 2011) assessed the effects of long-term aerobic exercise program on RT. Subjects performed 15 minutes of moderate intensity exercise on a cycle ergometer every other day for three months; as a result, RT significantly improved. Similarly, Asamoah et al. (Asamoah et al., 2013) determined that after a 6 week ( 3 sessions per week) aerobic training intervention, processing speed significantly decreased and was accompanied by improvements in aerobic capacity. These findings, in addition to others (Etnier, Nowell, Landers, \& Sibley, 2006; Kamijo, Nishihira, Higashiura, \& Kuroiwa, 2007; Ludyga, Gerber, Pühse, Looser, \& Kamijo, 2020), suggest that higher levels of cardiorespiratory fitness are intrinsically linked to faster RT (Herold, Törpel, Schega, \& Müller, 2019; Kamijo et al., 2007).

Although most previous research has examined the relationship between aerobic exercise and cognitive function, there is still evidence that muscular fitness (resistance) training, which targets improvements in both muscular strength and endurance, can positively stimulate the brain. It is believed to improve cognitive function through enhanced neurotransmitter activity, blood flow and central nervous system function (Herold et al., 2019; Wilke et al., 2019). For example, Frith and Loprinzi (2018) evaluated the association between lower extremity muscular strength and cognitive function; muscular strength, measured as maximal isokinetic contraction of the leg, was significantly associated with improved executive cognitive function. Additionally, a meta-analysis (Colcombe \& Kramer, 2003) indicated that long-term resistance training programs significantly improve executive functions related to speed-related tasks, supporting the claim that muscular fitness training can also augment cognitive function.

Few studies have investigated physical fitness profiles of eSports gamers specifically (Kari \& Karhulahti, 2016; Kari et al., 2019), but they have suggested that including physical activity into their training programs could potentially have a positive effect on eSports performance. However, this relationship has yet to be explored. The connection between health-related physical fitness components and RT in eSports gamers is not well understood. Therefore, the purpose of this study was to examine the relationship between the five essential components of physical fitness (i.e., body composition, cardiorespiratory fitness, muscular endurance, muscular strength, and flexibility) and RT 
in eSports gamers. The researchers hypothesized that cardiorespiratory fitness and muscular fitness would be significantly correlated with RT, as aerobic and resistance exercise have been suggested to augment cognitive function.

\section{METHODS}

\section{Experimental Approach to the Problem}

The experimental approach of this study consisted of comparing all five essential components of physical fitness to mean reaction time in eSports gamers. Each subject reported to the laboratory for three visits. Subjects were required to complete all three laboratory visits within a two-week period, with at least 24 hours between each visit. The first visit consisted of fitness testing. The second visit was a familiarization with the cognitive task i.e., Stroop Color Word Task. Subjects completed the Stroop Color Word task, again, at their third and final visit; results from this visit were used for analysis.

\section{Subjects}

The study included 27 individuals [all male, body mass index (BMI) $25.6 \pm 4.4 \mathrm{~kg} \cdot \mathrm{m}^{-2}$, ages $21.2 \pm$ 3.3 years; mean $\pm \mathrm{SD}$ ] who reported gaming at least five hours per week (weekly average $15.6 \pm 8.5$ hours) for the preceding three months. Subjects were recruited from the eSports club and intramural teams at a Midwestern university. A Physical Activity Readiness Questionnaire (PAR-Q+) was completed prior to participating in the study to screen individuals for symptoms or medical conditions that would contraindicate exercise. Individuals who sustained any musculoskeletal injury within the last six months were excluded from participation. The study was approved by the university's Human Subjects Institutional Review Board. Once subjects met all inclusion criteria, they read and signed an informed consent.

\section{Procedure}

During the first visit, subjects completed the PAR-Q+ and were asked to read and sign the informed consent document. Anthropometric measures (height and weight) were recorded, and subjects were familiarized with laboratory equipment and all experimental protocols. Prior to completing any tests, the Borg 6-20 rating of perceived exertion (RPE) scale (Figure 1) (Borg, 1998) was described to subjects in detail; subjects were informed of the scale values and the corresponding descriptors. Subjects were then asked to practice exercising at each specified RPE on a cycle ergometer to ensure full understanding of the scale. Subjects were then asked to complete five exercise tasks to test for all components of health-related physical fitness. The order of testing followed the American College of Sports Medicine (ACSM) guidelines and is listed accordingly below (ACSM, 2017).

\section{Visit 1: Fitness Testing}

Body Composition

First, body composition was assessed using an In-Body 570 body composition scale (Hangzhou, China). Subjects were asked to wipe their hands and feet with tissue provided by the manufacturer to enhance electrical conductivity. Thereafter, they were asked to stand barefoot on the scale and follow the prompts (e.g., holding or not holding the handles) while the scale measured body composition. Total body fat percentage (BF\%) was recorded.

\section{Cardiorespiratory Fitness}

Subjects were then fitted with a Polar chest strap heart rate monitor (Polar H10, USA) and instructed to complete a five-minute warm-up on a cycle ergometer (Wattbike Pro, Woodway, UK) at a selfselected intensity. Subjects completed a submaximal protocol, which was a modified version of the self-paced $\mathrm{VO}_{2} \max (\mathrm{SPV})$ test (Faulkner, Mauger, Woolley, \& Lambrick, 2015). The test was 


\begin{tabular}{|c|c|}
\hline Rating & Perceived Exertion \\
\hline 6 & No exertion \\
\hline 7 & Extremely light \\
\hline 8 & \\
\hline 9 & Very light \\
\hline 10 & \\
\hline 11 & Light \\
\hline 12 & \\
\hline 13 & Somewhat hard \\
\hline 14 & \\
\hline 15 & Hard \\
\hline 16 & \\
\hline 17 & Very hard \\
\hline 18 & \\
\hline 19 & Extremely hard \\
\hline 20 & Maximal exertion \\
\hline
\end{tabular}

exactly eight minutes in length, separated into four, two-minute stages. Each stage was perceptually regulated using RPE values of 11 (fairly light), 13 (somewhat hard), 15 (hard), and 17 (very hard), in that exact order. In order to maintain the prescribed RPE for each stage, subjects were allowed to adjust their cadence and resistance for the duration of the test. Upon completion of the test, subjects cooled down at a self-selected intensity for a minimum of five minutes.

Using a TrueOne 2400 metabolic cart (ParvoMedics, Sandy, UT), all expired gases during the submaximal test were collected. Flow and gas calibrations were performed 5-10 minutes prior to each test to ensure accurate recording. Fifteen-breath rolling averages were used (Robergs, Dwyer, \& Astorino, 2010) to determine maximal values for relative oxygen consumption $\left(\mathrm{VO}_{2}\right)$, carbon dioxide production $\left(\mathrm{VCO}_{2}\right)$, respiratory exchange ratio (RER), ventilation (VE), and heart rate (HR).

Because a submaximal test was utilized, it was necessary to calculate the estimated $\mathrm{VO}_{2}$ max. First, the age-predicted maximal heart rate (HRmax) formula (220 - age) was used to determine the predicted HRmax. Then, for the last 15 seconds of each stage of the SPV, HR and $\mathrm{VO}_{2}$ values were 
Figure 2. Prediction of $\mathrm{VO}_{2}$ max for an exemplary subject. Best fit line of the first four data points was used to determine the regression equation. Then, this line was extrapolated to the predicted $\mathrm{HR}_{\max }\left((200 \mathrm{bpm})\right.$ and predicted $\mathrm{VO}_{2} \max \left(39.37 \mathrm{~m} \times \mathrm{kg}^{-1} \times \mathrm{min}^{-1}\right)$.

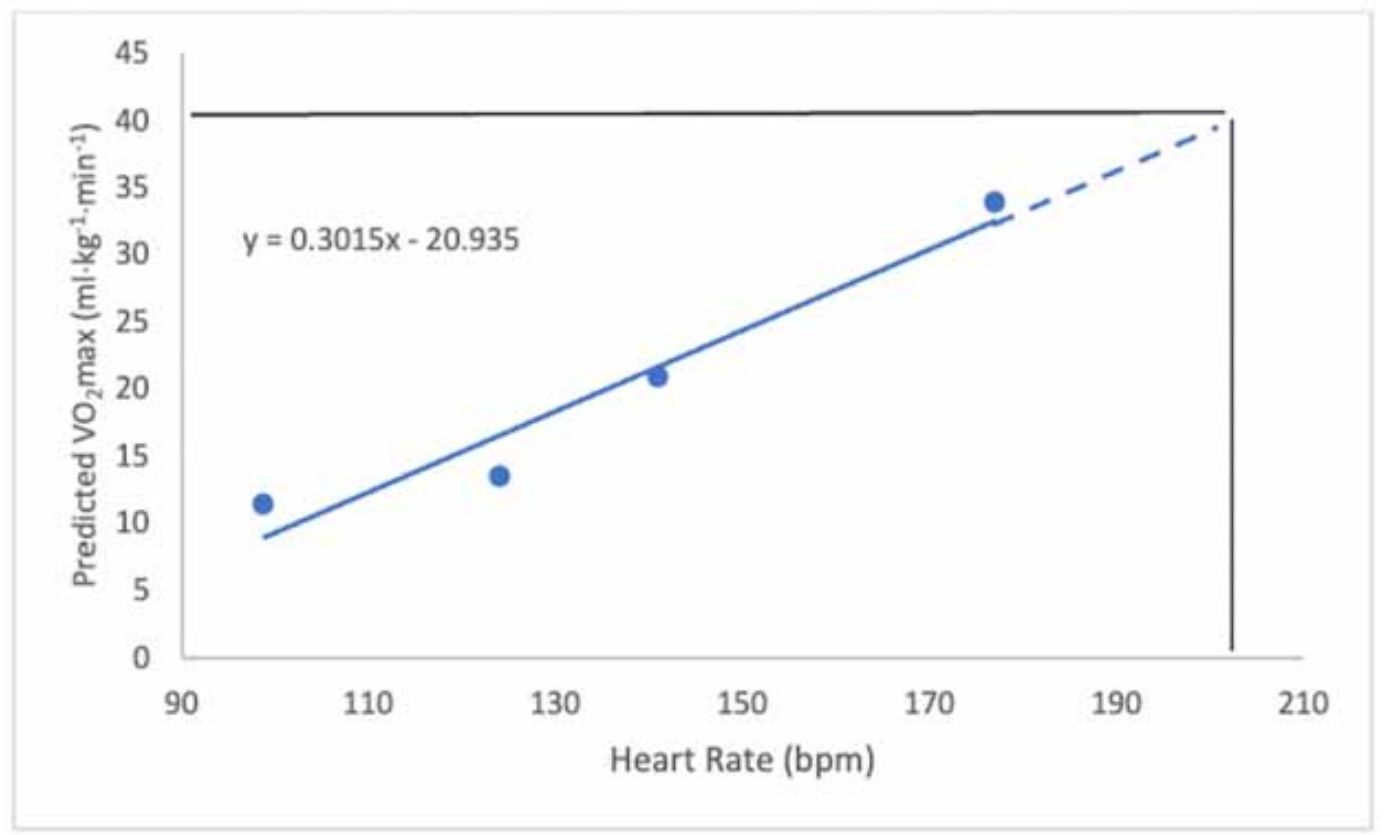

averaged; these values were then plotted against each other (for each stage) in order to create a best fit line used to obtain a regression equation and determine the slope of the line. The constant from the regression equation (b), the subject's calculated HRmax (x) and the computed slope (m) were input into the linear regression equation to solve for predicted $\mathrm{VO}_{2} \max$ (Figure 2).

\section{Muscular Endurance}

Subjects were given adequate time to rest (approximately 10 minutes) before implementing a simple push-up test. Subjects were instructed to start in the standard "down" position (hands pointing forward and aligned under shoulders, back straight, head up, using toes as the pivot point). All subjects were instructed to raise their body by straightening their elbows, and then returned to a "down" position until their chin touched the mat (chest approximately one inch from the mat). The maximum number of repetitions completed consecutively, without rest, were counted; if the subject failed to overcome fatigue or maintain the appropriate technique within two repetitions, the test was stopped.

\section{Muscular Strength}

Subjects were given approximately 10 minutes to rest before completing a 1-repetition maximum (1RM) leg press. Subjects completed a warm-up by completing 10 submaximal repetitions on the leg press. Thereafter, an initial weight was chosen that was between $50 \%-70 \%$ of the subject's perceived maximal capacity. Resistance was progressively increased by $2.5-20 \mathrm{~kg}$ until the subject could only implement one repetition with full range of motion and proper form; this was recorded as their 1-RM 
and was determined within a maximum of four trials. Additionally, to ensure proper form and safety, two researchers were present during each maximal test.

\section{Flexibility}

The Canadian Trunk Forward Flexion test was used to assess flexibility. Subjects were allowed to stretch for approximately five minutes before completing the test. They removed their shoes and sat on the ground, placing their feet flat against a sit-and-reach box at the 26-centimeter mark positioning inner edges of the soles approximately six inches apart. Subjects were instructed to slowly reach forward (no fast, jerky movements) as far as possible, holding their position for approximately two seconds. Researchers ensured the subjects exhaled as they reached forward, with hands overlapped and knees fully extended. Three trials were completed, and the maximum value was recorded.

\section{Visits 2 and 3: Cognitive Testing}

Subjects then visited the lab for a second time and were taken through a familiarization trial of the cognitive test, the Stroop Task. A full familiarization was implemented to ensure reliable and accurate test indices. During the third visit, subjects completed the Stroop Task again and data from this visit was used for analyses.

\section{Stroop Task}

Subjects were asked to complete the Stroop Color Word Task (Franzen, Tishelman, Sharp, \& Friedman, 1987) (Millisecond Software, Seattle, WA) to measure RT. Subjects were seated comfortably in a chair with a laptop computer placed on a desk in front of them. Subjects were shown color words (i.e., red, green, blue, black), which were each written in a color. They were asked to indicate the color the word was typed in (not the meaning of the word) by pressing a specific key ( $\mathrm{D}=$ red, $\mathrm{F}=$ green, $\mathrm{J}=$ blue, $\mathrm{K}=$ black) as fast as they could, with as few errors as possible. RT for congruent, incongruent and control trials was recorded. Congruent trials are those in which the written word is the same as the font color of the word presented (e.g. the word "red" written in red colored font); incongruent trials are when the word is presented in a colored font that is not the same as the written word (e.g. "red" written in green colored font); control, or neutral trials are when a solid block of color appears (Figure 3). The mean RT is the average of these three trials. The percentage of correct responses for congruent, incongruent and control were also collected. Lastly, the inverse efficiency score (IES) was calculated (i.e. RT divided by percent correct) for congruent, incongruent and control trials in order to create an observational measure that combines speed and accuracy (Bruyer \& Brysbaert, 2011).

\section{Statistical Analysis}

All data was analyzed using IBM SPSS Statistics (Version 25, Armonk, NY). Descriptive statistics were used for all variables. A bivariate Pearson correlation was used to determine the relationship between the five components of physical fitness and mean RT. The Pearson correlation coefficient, $r$, was used to determine the strength of the relationship. Significance was set a priori $\mathrm{p}<.05$.

\section{RESULTS}

Descriptive characteristics of subjects can be found in Table 1.

Results of the bivariate Pearson correlation indicated a significant negative relationship between mean RT and cardiorespiratory fitness $(r=-.338, \mathrm{p}=.043)$. Additionally, IES was significantly related to cardiorespiratory fitness for incongruent $(r=-.373, \mathrm{p}=.028$; Figure 4$)$ and control trials $(r=-.333$, $\mathrm{p}=.045)$. There were no correlations between mean RT and body composition $(r=.149, \mathrm{p}=.229)$, muscular endurance $(r=-.160, \mathrm{p}=.212)$, muscular strength $(r=-.020 \mathrm{p}=.461)$ or flexibility $(r=-.030$, 
Figure 3. The Stroop Task. Subjects were asked to identify the color of the word presented to them using a corresponding button on a keyboard $(D=$ red, $F=$ green, $I=$ blue, $K=$ black). A congruent trial is when the font color is the same as the word meaning. An incongruent trial is when the font color is different than the word meaning. A control trial is when a solid block of color appears.
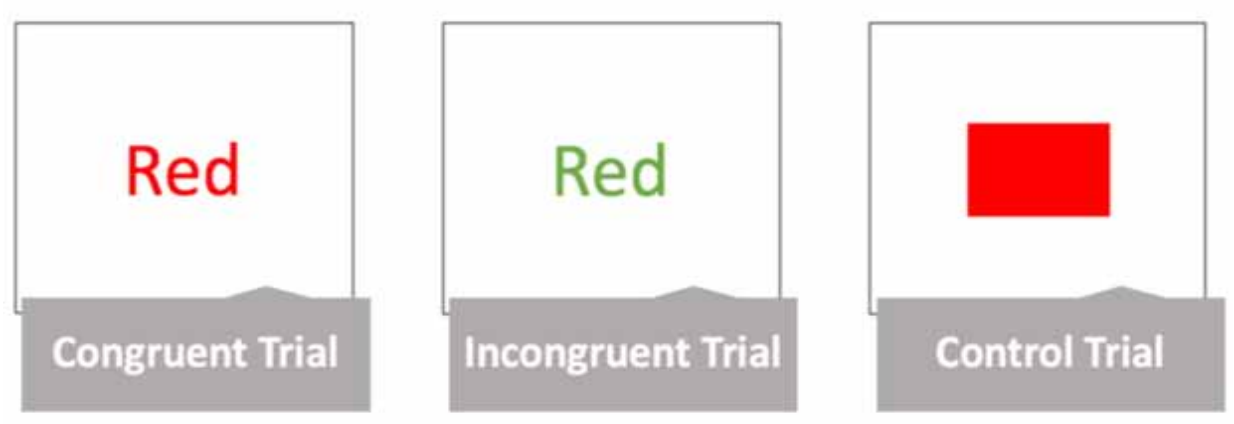

Table 1. Descriptive characteristics of subjects

\begin{tabular}{|c|c|c|}
\hline Variable & Mean & SD \\
\hline Age (years) & 21.2 & 3.3 \\
\hline $\mathrm{BMI}\left(\mathrm{kg} \times \mathrm{m}^{-2}\right)$ & 25.6 & 4.3 \\
\hline Body Fat (\%) & 16.5 & 8.3 \\
\hline $\mathrm{VO}_{2} \max \left(\mathrm{ml} \times \mathrm{kg}^{-1} \times \mathrm{min}^{-1}\right)$ & 45.7 & 11.8 \\
\hline Push-Ups (number completed) & 30.1 & 14.0 \\
\hline 1-Repetition maximum (kg) & 220.9 & 66.7 \\
\hline Flexibility $(\mathrm{cm})$ & 22.4 & 9.3 \\
\hline
\end{tabular}

$\mathrm{p}=.441)$. Average RT and response accuracy for congruent, incongruent and control trials are shown in Table 2.

\section{DISCUSSION}

The main purpose of this study was to examine the relationship between the five essential components of physical fitness and RT in eSports gamers. To their author's knowledge, this is the first time 
Table 2. Average reaction time and accuracy for Stroop Task trials.

\begin{tabular}{|l|l|l|}
\hline Trial & Reaction Time (ms) & Accuracy (\%) \\
\hline Congruent & $667.7 \pm 93.9$ & $95.9 \pm 5.2$ \\
\hline Incongruent & $761.2 \pm 165.0$ & $94.7 \pm 5.2$ \\
\hline Control & $681.5 \pm 119.0$ & $96.0 \pm 4.9$ \\
\hline
\end{tabular}

Note: values presented as mean \pm SD.

Figure 4. Relationship between incongruent IES and predicated $\mathrm{VO}_{2} \max$

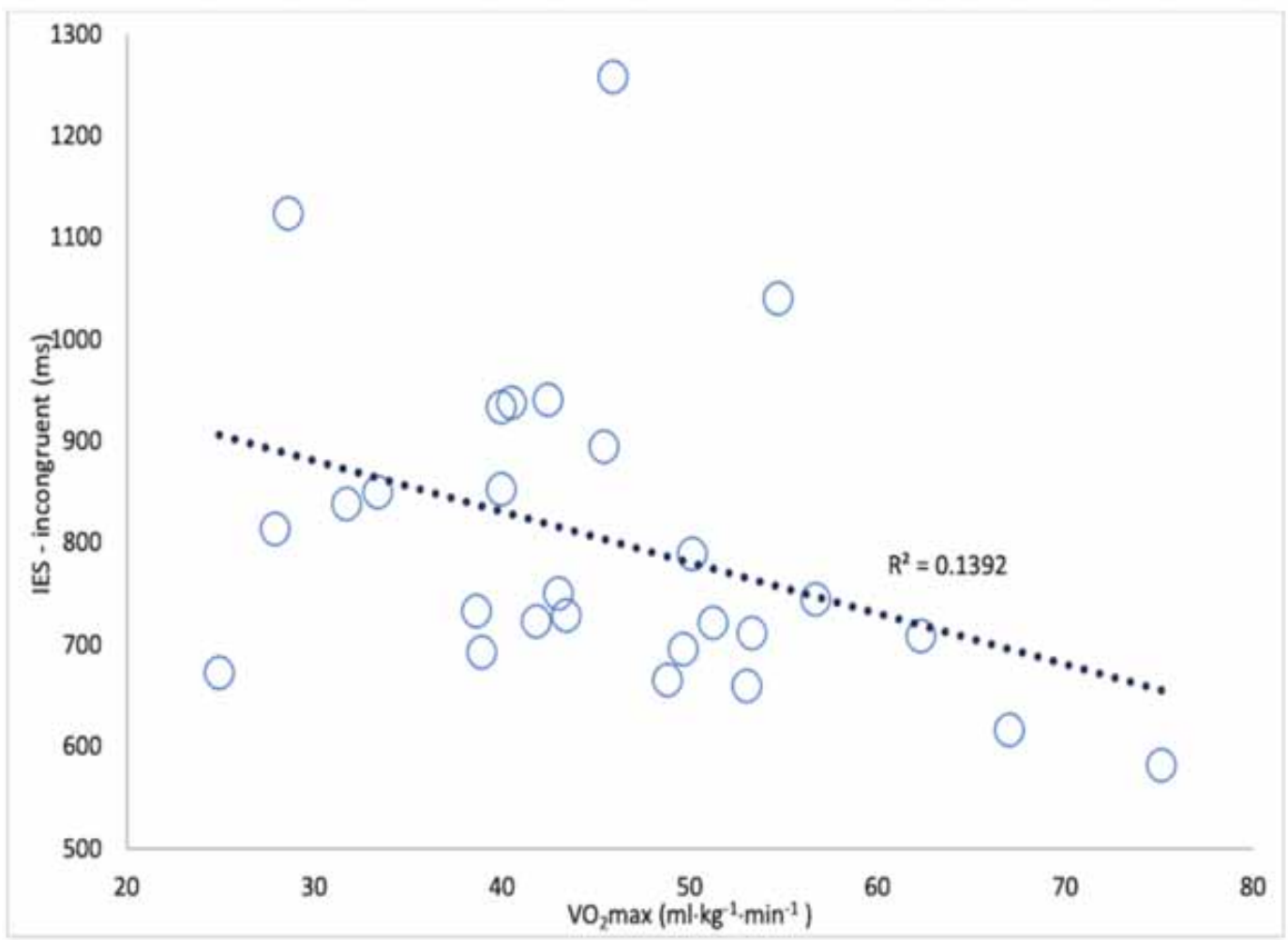

this relationship has been assessed using eSports gamers as the subject group. The hypothesis was somewhat supported; there was a significant correlation between RT and cardiorespiratory fitness. However, RT was not correlated with body composition, muscular endurance, muscular strength, or flexibility. The results suggest that an increased $\mathrm{VO}_{2}$ max can augment cognitive function, which supports exercise programs that enhance aerobic power as a potential method to improve eSports gaming performance.

A substantial amount of research has supported an unequivocal relationship between cardiorespiratory fitness and overall health benefits. Moreover, higher levels of cardiorespiratory fitness are associated with a decreased risk of cardiovascular disease and all-cause mortality (Bherer, Erickson, \& Liu-Ambrose, 2013). Currently, aerobic exercise is considered not only to be a preventative measure of these risks, but a means of enhancing cognitive function, thus providing a 
reason for eSports gamers to highly benefit from it. The results from the current study are corroborated by previous studies suggesting that consistent aerobic exercise is able to improve RT. Renaud et al. (Renaud, Maquestiaux, Joncas, Kergoat, \& Bherer, 2010) suggesting that a three month aerobic training program (one hour sessions, three days per week) significantly improved RT. Dustman et al. (Dustman et al., 1984) investigated the effects of a four month, aerobic training program (one hour sessions, three days per week) on Stroop Task performance and reported similar results. Another study by Smiley-Oyen et al. (Smiley-Oyen, Lowry, Francois, Kohut, \& Ekkekakis, 2008) indicated that after 5 months of aerobic training (30 minute sessions, three days per week), response accuracy on the Stroop test improved, while after an additional 5 months, response accuracy continued to improve and response speed significantly increased. While eSports gaming seems to promote a more sedentary lifestyle (Polman et al., 2018), health professionals can use this information to influence and motivate gamers towards engaging in frequent aerobic training to potentially improve gaming performance with the added benefit of improving health parameters.

Although a relationship between body composition (\%BF) and RT was not established, there is a significant relationship between unhealthy weight gain (or increased \%BF) and increased risk for cardiovascular disease risk factors and associated health issues (ACSM, 2017). However, these risks may be lowered by increasing cardiorespiratory fitness (ACSM, 2017). This, again, highlights the importance of training to improve aerobic power. All subjects in the current study were classified as young-adults (ages 18-35 years), which is a population that has the highest rate of weight gain, and therefore a greater risk of lifestyle-related health conditions. In fact, of the 27 subjects, 14 fell within the "poor" or "very poor" cardiorespiratory fitness classifications per ACSM normative values (ACSM, 2017), while only three were considered "excellent" or "superior" for their age group. There is a critical need for health professionals to step in and educate eSports gamers on the importance of improving cardiorespiratory fitness. Additionally, health professionals should express the additional benefit that increased aerobic power augments cognitive function; therefore, eSports gamers can gain insight on how cognitive enhancement translates to enhancing eSport performance.

Previous research has suggested that RT has improved after partaking in an acute bout of resistance training. For example, Chang et al. (Chang \& Etnier, 2009) determined that after completing six exercises (two sets of each, with 10 repetitions), speed of processing for the Stroop Color Word Task was increased as shown by a faster reaction time. In a recent meta-analysis (Wilke et al., 2019), acute bouts of resistance exercise were indicated to enhance cognitive function in healthy adults (age range 20.4-72.3 years). In regard to long-term resistance training, Coetsee and Terblanche (Coetsee $\&$ Terblanche, 2017) investigated the effects of a 16-week (three days per week) resistance training program on RT measured by the Stroop Task. Subjects were inactive adults between ages 55-75 years; RT significantly improved from pre- to post-training. Liu-Ambrose et al. (Liu-Ambrose, Nagamatsu, Voss, Khan, \& Handy, 2012) determined that a 12-month, two day per week resistance training program improved performance on tasks that test executive function (e.g. Stroop Task) in women between the ages of 65 and 75 years. In the current study, there was a weak relationship between both components of muscular fitness and RT. It is difficult to determine the reason for this non-significant relationship. It is worth mentioning that the current study used young, healthy individuals with fairly high levels of muscular fitness. Per ACSM normative values (ACSM, 2017), over $80 \%$ of the subjects were "well-above average" for muscular strength, and more than $70 \%$ of subjects were "good (40\%)," "very good (20\%)," or "excellent (40\%)" for muscular endurance. Due to this homogenous sample, there is a strong possibility that a lack of variability within these variables is the reason for the lack of a significant relationship to reaction time. Regardless, future research is warranted that includes long-term resistance training programs and is more inclusive of younger populations (e.g., children and young adults between the ages of 13-35 years) as most eSports gamers fall within these populations.

During eSports game play, there is a copious amount of visual and auditory stimuli. Frequently, eSports gamers have to selectively decide which stimuli are to be focused on and which can be disregarded as less relevant when striving to perform optimally (Donohue, Woldorff, \& Mitroff, 2010). 
The Selective Attention Theory states that the brain has an ability to grant certain stimuli an increased rate of processing, while virtually ignoring others (Wright, 2017). During the Stroop Task, selective visual attention is measured; specifically with incongruent trials, the brain must decide whether the text or the color is more important (Wright, 2017). Incongruent tasks require higher selective attention and greater cognitive processing (Rosner, D’Angelo, MacLellan, \& Milliken, 2015). In the current study, IES for congruent scores were significantly related to cardiorespiratory fitness, thus proposing that a greater aerobic capacity correlates to quicker and more accurate responses. Greater selective attention can strongly impact eSports gamers, so it can be hypothesized that improving cardiorespiratory fitness could present benefits to this population.

One major public health concern is sedentary behavior, which has been highly associated with gaming for decades. However, the stigma of gamers embodying a physically inactive lifestyle has been disputed numerous times. A 2016 survey reported that the over $80 \%$ of eSports gamers participate in an average of 1.08 hours of physical activity daily (Kari \& Karhulahti, 2016). Although eSports gamers are required to "train" for their sport, like any other professional athlete, training does not inhibit the implementation of physical exercise (Kari \& Karhulahti, 2016). Stereotyping gamers has led to false impressions about eSports (Kari \& Karhulahti, 2016). Health professionals should work together with eSports gamers to publicize training regimes they undergo in order to inspire physically active lifestyles and more positively portray the gaming realm. With respect to the significant number of individuals playing and/or spectating eSports, there is opportunity to use this population as a way to influence many positive health and lifestyle choices and public health issues (Polman et al., 2018). Future researchers should focus on how various exercise prescriptions benefit eSports gamers with regard to physical and cognitive skills.

In conclusion, the current study reveals that cardiorespiratory fitness and RT are significantly correlated in eSports gamers. The novel finding is that an increased aerobic capacity augments IES scores, suggesting that greater cardiorespiratory fitness is correlated to quicker and more accurate responses. This provides further support for consistent physical activity (that improves aerobic power) to benefit acute and long-term health with additional benefit of enhancing eSports gaming performance. The authors highly suggest that competitive eSports teams consider hiring exercise physiologists or personal trainers who are able to construct appropriate exercise prescriptions and training programs to optimize aerobic capacity.

\section{LIMITATIONS}

While participants in this study were all male, college-aged individuals, the authors would like to note that participation was open to both males and females between the ages of 18-35 years. The demographics of the final recruitment sample was not surprising, as many eSports studies have predominantly used male college-aged participants. Future research could benefit from using different recruiting methods to specifically target female participants and see if sex-related differences are present.

While it would be useful to examine "eSports performance" using actual game play, this was unfortunately not possible. Since there are so many gaming types (e.g., Multiplayer Online Battle Arena, First Person Shooter, Battle Royale, etc.) there is no one, ideal testing measure that specifically determines "eSports performance." Consequently, an effort was made to assess an identifiable measure (i.e., reaction time) that is universally paramount to all gaming types.

\section{PRACTICAL APPLICATION}

Findings from this study could be useful for eSports gamers, their respective health coaches, or exercise physiologists. The researchers suggested that there was a correlation between cardiorespiratory fitness and mean reaction time. Additionally, IES for congruent scores were significantly related to 
cardiorespiratory fitness, suggesting that a greater aerobic capacity not only correlates to quicker reaction time, but more accurate responses. As a result, when creating exercise prescriptions for eSports gamers, health coaches should consider including workouts that target increasing aerobic power. Incorporating aerobic-based training, aimed to increased $\mathrm{VO}_{2} \max$, into eSport gamer regimes could be favorable, as it has the potential to improve cognitive function and enhance gaming performance in competitive events. Additionally, due to the significant number of individuals playing and spectating eSports gamers and events, there is great opportunity to use this population e.g., eSports gamers, as a way to influence many positive health and lifestyle choices.

This research received no specific grant from any funding agency in the public, commercial, or not-for-profit sectors. 


\section{REFERENCES}

ACSM. (2017). American College of Sports Medicine's Exercise Testing and Prescription. Lippincott Williams \& Wilkins.

Asamoah, S., Siegler, J., Chang, D., Scholey, A., Yeung, A., \& Cheema, B. S. (2013). Effect of aerobic training on cognitive function and arterial stiffness in sedentary young adults: A pilot randomized controlled trial. Physiology Journal, 2013, 1-9. doi:10.1155/2013/847325

Bányai, F., Griffiths, M., Király, O., \& Demetrovics, Z. (2019). The psychology of esports: A systematic literature review. Journal of Gambling Studies, 35(2), 351-365. doi:10.1007/s10899-018-9763-1 PMID:29508260

Bediou, B., Adams, D., Mayer, R., Tipton, E., Green, C., \& Bavelier, D. (2018). Meta-analysis of action video game impact on perceptual, attentional, and cognitive skills. Psychological Bulletin Journal, 144(1), 77-110. doi:10.1037/bul0000130 PMID:29172564

Bherer, L., Erickson, K., \& Liu-Ambrose, T. (2013). A review of the effects of physical activity and exercise on cognitive and brain functions in older adults. Journal of Aging Research, 2013, 1-8. doi:10.1155/2013/657508 PMID:24102028

Borg, G. (1998). Borg's Perceived Exertion and Pain Scales. Human Kinetics.

Bruyer, R., \& Brysbaert, M. (2011). Combining speed and accuracy in cognitive psychology: Is the inverse efficiency score (IES) a better dependent variable than the mean reaction time (RT) and the percentage of errors (PE)? Psychologica Belgica, 51(1), 5-13. doi:10.5334/pb-51-1-5

Campbell, M., Toth, A., Moran, A., Kowal, M., \& Exton, C. (2018). eSports: A new window on neurocognitive expertise? Progress in Brain Research, 240, 161-174. doi:10.1016/bs.pbr.2018.09.006 PMID:30390829

Chang, Y.-K., \& Etnier, J. L. (2009). Effects of an acute bout of localized resistance exercise on cognitive performance in middle-aged adults: A randomized controlled trial study. Psychology of Sport and Exercise, 10(1), 19-24. doi:10.1016/j.psychsport.2008.05.004

Coetsee, C., \& Terblanche, E. (2017). Cerebral oxygenation during cortical activation: The differential influence of three exercise training modalities. A randomized controlled trial. European Journal of Applied Physiology, 117(8), 1617-1627. doi:10.1007/s00421-017-3651-8 PMID:28567669

Colcombe, S., \& Kramer, A. F. (2003). Fitness effects on the cognitive function of older adults: A meta-analytic study. Journal of Psychological Science, 14(2), 125-130. doi:10.1111/1467-9280.t01-1-01430 PMID:12661673

Collins, L., \& Long, C. (1996). Visual reaction time and its relationship to neuropsychological test performance. Archives of Clinical Neuropsychology, 11(7), 613-623. doi:10.1093/arclin/11.7.613 PMID:14588445

Donohue, S. E., Woldorff, M. G., \& Mitroff, S. R. (2010). Video game players show more precise multisensory temporal processing abilities. Attention, Perception \& Psychophysics, 72(4), 1120-1129. doi:10.3758/ APP.72.4.1120 PMID:20436205

Dustman, R. E., Ruhling, R. O., Russell, E. M., Shearer, D. E., Bonekat, H. W., Shigeoka, J. W., Wood, J. S., \& Bradford, D. C. (1984). Aerobic exercise training and improved neuropsychological function of older individuals. Neurobiology of Aging, 5(1), 35-42. doi:10.1016/0197-4580(84)90083-6 PMID:6738784

Dye, M. W., Green, C., \& Bavelier, D. (2009). Increasing speed of processing with action video games. Current Directions in Psychological Science, 18(6), 321-326. doi:10.1111/j.1467-8721.2009.01660.x PMID:20485453

Etnier, J. L., Nowell, P. M., Landers, D. M., \& Sibley, B. A. (2006). A meta-regression to examine the relationship between aerobic fitness and cognitive performance. Brain Research. Brain Research Reviews, 52(1), 119-130. doi:10.1016/j.brainresrev.2006.01.002 PMID:16490256

Faulkner, J., Mauger, A., Woolley, B., \& Lambrick, D. (2015). The efficacy of a self-paced VO2max test during motorized treadmill exercise. International Journal of Sports Physiology and Performance, 10(1), 99-105. doi:10.1123/ijspp.2014-0052 PMID:24912200 
Franzen, M. D., Tishelman, A. C., Sharp, B. H., \& Friedman, A. (1987). An investigation of the test-retest reliability of the stroop colorword test across two intervals. Archives of Clinical Neuropsychology, 2(3), $265-272$. doi:10.1093/arclin/2.3.265 PMID:14589618

Frith, E., \& Loprinzi, P. (2018). The association between lower extremity muscular strength and cognitive function in a national sample of older adults. Journal of Lifestyle Medicine, 8(2), 99-104. doi:10.15280/jlm.2018.8.2.99 PMID:30474005

Hallmann, K., \& Giel, T. (2018). eSports-Competitive sports or recreational activity? Sport Management Review, 21(1), 14-20. doi:10.1016/j.smr.2017.07.011

Hamari, J., \& Sjöblom, M. (2017). What is eSports and why do people watch it? Internet Research, 27(2), 211-232. doi:10.1108/IntR-04-2016-0085

Herold, F., Törpel, A., Schega, L., \& Müller, N. (2019). Functional and/or structural brain changes in response to resistance exercises and resistance training lead to cognitive improvements-a systematic review. European Review of Aging and Physical Activity, 16(1), 10. doi:10.1186/s11556-019-0217-2 PMID:31333805

Kamijo, K., Nishihira, Y., Higashiura, T., \& Kuroiwa, K. (2007). The interactive effect of exercise intensity and task difficulty on human cognitive processing. International Journal of Psychophysiology, 65(2), $114-121$. doi:10.1016/j.ijpsycho.2007.04.001 PMID:17482699

Kari, T., \& Karhulahti, V.-M. (2016). Do E-athletes move?: A study on training and physical exercise in elite E-Sports. International Journal of Gaming and Computer-Mediated Simulations, 8(4), 53-66. doi:10.4018/ IJGCMS.2016100104

Kari, T., Siutila, M., \& Karhulahti, V.-M. (2019). An extended study on training and physical exercise in esports. In Exploring the Cognitive, Social, Cultural, and Psychological Aspects of Gaming and Simulations (pp. 270292). IGI Global. doi:10.4018/978-1-5225-7461-3.ch010

Liu-Ambrose, T., Nagamatsu, L. S., Voss, M. W., Khan, K. M., \& Handy, T. C. (2012). Resistance training and functional plasticity of the aging brain: A 12-month randomized controlled trial. Neurobiology of Aging, 33(8), 1690-1698. doi:10.1016/j.neurobiolaging.2011.05.010 PMID:21741129

Ludyga, S., Gerber, M., Pühse, U., Looser, V. N., \& Kamijo, K. (2020). Systematic review and meta-analysis investigating moderators of long-term effects of exercise on cognition in healthy individuals. Nature Human Behaviour, 4(6), 1-10. doi:10.1038/s41562-020-0851-8 PMID:32231280

Nagorsky, E., \& Wiemeyer, J. (2020). The structure of performance and training in esports. PLoS One, 15(8), e0237584. doi:10.1371/journal.pone.0237584 PMID:32841263

Pallavicini, F., Ferrari, A., \& Mantovani, F. (2018). Video games for well-being: A systematic review on the application of computer games for cognitive and emotional training in the adult population. Frontiers in Psychology, 9, 2127. doi:10.3389/fpsyg.2018.02127 PMID:30464753

Perrin, T., Faure, C., Nay, K., Cattozzo, G., Sorel, A., Kulpa, R., \& Kerhervé, H. (2019). Virtual Reality Gaming Elevates Heart Rate but Not Energy Expenditure Compared to Conventional Exercise in Adult Males. International Journal of Environmental Research and Public Health, 16(22), 4406. doi:10.3390/ijerph16224406 PMID:31717971

Polman, R., Trotter, M., Poulus, D., \& Borkoles, E. (2018). eSport: Friend or Foe? Paper presented at the Joint International Conference on Serious Games. doi:10.1007/978-3-030-02762-9_1

Radák, Z. (2018). The Physiology of Physical Training. Academic Press.

Renaud, M., Maquestiaux, F., Joncas, S., Kergoat, M.-J., \& Bherer, L. (2010). The effect of three months of aerobic training on response preparation in older adults. Frontiers in Aging Neuroscience, 2, 148. doi:10.3389/ fnagi.2010.00148 PMID:21151355

Robergs, R. A., Dwyer, D., \& Astorino, T. (2010). Recommendations for improved data processing from expired gas analysis indirect calorimetry. The Journal of Sports Medicine, 40(2), 95-111. doi:10.2165/11319670000000000-00000 PMID:20092364 
Rosner, T. M., D’Angelo, M. C., MacLellan, E., \& Milliken, B. (2015). Selective attention and recognition: Effects of congruency on episodic learning. Psychological Research, 79(3), 411-424. doi:10.1007/s00426-0140572-6 PMID:24859839

Seo, Y., \& Jung, S.-U. (2016). Beyond solitary play in computer games: The social practices of eSports. Journal of Consumer Culture, 16(3), 635-655. doi:10.1177/1469540514553711

Smiley-Oyen, A. L., Lowry, K. A., Francois, S. J., Kohut, M. L., \& Ekkekakis, P. (2008). Exercise, fitness, and neurocognitive function in older adults: The "selective improvement" and "cardiovascular fitness" hypotheses. Annals of Behavioral Medicine, 36(3), 280-291. doi:10.1007/s12160-008-9064-5 PMID:18825471

Smith, P. J., Blumenthal, J. A., Hoffman, B. M., Cooper, H., Strauman, T. A., Welsh-Bohmer, K., Browndyke, J. N., \& Sherwood, A. (2010). Aerobic exercise and neurocognitive performance: A meta-analytic review of randomized controlled trials. Psychosomatic Medicine, 72(3), 239-252. doi:10.1097/PSY.0b013e3181d14633 PMID:20223924

Verma, S., Mishra, A., \& Singh, A. (2011). Effect of long term physical exercise training on auditory and visual reaction time. Physiotherapy and Occupational Therapy Journal, 5(3), 126-129.

Wang, P., Liu, H.-H., Zhu, X.-T., Meng, T., Li, H.-J., \& Zuo, X.-N. (2016). Action video game training for healthy adults: A meta-analytic study. Frontiers in Psychology, 7, 907. doi:10.3389/fpsyg.2016.00907 PMID:27378996

Wilke, J., Giesche, F., Klier, K., Vogt, L., Herrmann, E., \& Banzer, W. (2019). Acute effects of resistance exercise on cognitive function in healthy adults: A systematic review with multilevel meta-analysis. The Journal of Sports Medicine, 49(6), 905-916. doi:10.1007/s40279-019-01085-x PMID:30838520

Wright, B. C. (2017). What Stroop tasks can tell us about selective attention from childhood to adulthood. British Journal of Psychology, 108(3), 583-607. doi:10.1111/bjop.12230 PMID:27786354

Rachel Dykstra completed her doctorate in Exercise Physiology at Western Michigan University. She is currently an Assistant Professor of Health and Human Performance at Nebraska Wesleyan University, teaching a variety of undergraduate courses including Basic Human Nutrition, Sport Nutrition, Exercise Testing \& Programming, and Research \& Statistical Methods in Exercise Science. Her research focuses on identifying variables that moderate the relationship between exercise and cognitive function.

Nicholas Hanson is an Associate Professor of Exercise Science at Western Michigan University. He received his doctorate in Kinesiology at the Ohio State University in 2013. His specific research focus is on learning more about how the brain and human perception influences performance under various conditions.

Panagiotis Koutakis received his PhD and post-doctoral training in Biomedical Sciences from the University of Nebraska at Omaha Medical Center in the Department of Surgery. He is currently an Assistant Professor at Baylor University and the Department of Biology, where his research focuses on the development of improved diagnostic and treatment methods for patients with peripheral artery disease. 